\title{
Análise das internações por condições sensíveis à Atenção Primária e a correlação dos gastos em saúde em 50 municípios de Minas Gerais
}

\author{
Analysis of hospitalizations due to sensitive conditions to Primary Care and the \\ consequences of health expenditures in 50 municipalities in Minas Gerais
}
Análisis de las hospitalizaciones por condiciones sensibles a la Atención Primaria y las consecuencias del gasto en salud en 50 municipios de Minas Gerais

Eduardo Gonçalves ${ }^{1}$, Fábio Luiz Mialhe ${ }^{1 *}$.

\begin{abstract}
RESUMO
Objetivo: Analisar possíveis associações entre as internações por condições sensíveis à atenção primária e os gastos em ações e serviços de saúde em 50 municípios do sul do estado de Minas Gerais. Métodos: Estudo transversal descritivo, utilizando-se de dados secundários de acesso am plo, para os anos de 2017e 2018. Os dados foram analisados por meio de modelos de regressão logística múltipla. Resultados: Os municípios apresentaram uma média de 23,1 e 22,7 internações por condições sensíveis em 2017 e 2018, respectivamente. Municípios com menor gasto com atividades de saúde em 2017, tiveram 3,16 vezes mais chance de apresentar maior número de internações por condições sensíveis em 2017 e 2018 ( $p=0,05)$. Entre os municípios com menor gasto com atividades de saúde em 2017,64,0\% tiveram mais internações por condições sensíveis em 2017 e 2018. Já entre aqueles com maior gasto, essa porcentagem foi menor $(36,0 \%)$. Conclusão: Maiores gastos em ações e serviços de saúde estiveram associados a menor número de internações por condições sensíveis à atenção primária.
\end{abstract}

Palavras-chave: Atenção primária à saúde, Gastos em saúde, Regionalização da saúde, Planejamento em saúde, Financiamento em saúde.

\section{ABSTRACT}

Objective: To analyze possible associations between hospitalizations for conditions sensitive to primary care and spending on health actions and services in 50 municipalities in the south of the state of Minas Gerais. Methods: Crosssectional descriptive study, using widely accessible secondary data for the years 2017 and 2018. Data were analyzed using statistical analysis of association. Results: On average, municipalities had 23.1 and 22.7 hospitalizations for sensitive conditions in 2017 and 2018, respectively. Municipalities with lower expenditure on health activities in 2017 were 3.16 times more likely to have a higher number of hospitalizations for sensitive conditions in 2017 and 2018 ( $p=0.05$ ). Among the municipalities with lower expenditure on health activitie s in $2017,64.0 \%$ had more hospitalizations for sensitive conditions in 2017 and 2018. Among those with higher expenditure, this percentage was lower (36.0\%). Conclusion: Higher spending on health actions and services were associated with a lower number of hospitalizations for conditions sensitive to primary care.

Keywords: Primary health care, Health expenditures, Regional health planning, Health planning, Healthcare financing.

\section{RESUMEN}

Objetivo: Analizar posibles asociaciones entre hospitalizaciones por condiciones sensibles a la atención primaria y gasto en acciones y servicios de salud en 50 municipios del sur del estado de Minas Gerais. Métodos: Estudio descriptivo transversal, utilizando datos secundarios am pliamente accesibles para los años 2017 y 2018. Los datos fueron analizados mediante análisis estadístico de asociación. Resultados: En promedio, los municipios tuvieron 23.1 y 22.7 hospitalizaciones por condiciones sensibles en 2017 y 2018, respectivamente. Los municipios con menor gasto en actividades de salud en 2017 tuvieron 3,16 veces más probabilidades de tener un mayor número de hospitalizaciones por condiciones sensibles en 2017 y 2018 ( $p=0,05)$. Entre los municipios con menor gasto en actividades de salud en 2017 , el $64,0 \%$ tuvo más hospitalizaciones por condiciones sensibles en 2017 y 2018 . Entre los de mayor gasto, este porcentaje fue menor $(36,0 \%)$. Conclusión: Un mayor gasto en acciones y servicios de salud se asoció con un menor número de hospitalizaciones por condiciones sensibles a la atención primaria.

Palabras clave: Atención primaria de salud, Gastos en salud, Regionalización, Planificación en salud, Financiación de la atención de la salud.

\footnotetext{
${ }^{1}$ Faculdade de Odontologia de Piracicaba (FOP), Universidade Estadual de Campinas (Unicamp), Piracicaba - SP.

*E-mail:mialhe@unicamp.br
} 


\section{INTRODUÇÃO}

Considerado como um sistema de saúde de sucesso na América Latina, para Santos HLPC, et al. (2021), - Sistema Único de Saúde (SUS) apresenta grandes desafios a serem superados. Dentre estes desafios a insuficiência de recursos para a manutenção deste direito social conquistado constitucionalmente, bem como um cenário de sucessivas crises econômicas aliadas a medidas de austeridade fiscal, os quais impactam na sobrevida e consolidação do sistema (MENDES A e FUNCIA FR, 2016).

No SUS, as redes de atenção à saúde são identificadas e representadas por meio de diversos pontos de atenção à saúde, cada qual com suas respectivas competências e serviços em localidades distintas, onde, nestas redes, a responsabilização sobre o centro de comunicação é atribuída à Atenção Primária à Saúde (APS) (UNIVERSIDADE FEDERAL DO MARANHÃO (UFMA), 2016

De acordo com Starfield B, et al. (2002) os atributos essenciais da APS, como o acesso de primeiro contato, longitudinalidade, integralidade e coordenação e, também os atributos derivados, expressos na orientação familiar, orientação comunitária e competência cultural são conceitos que se relacionam com a vocação da assistência neste nível de atenção aos usuários.

No contexto brasileiro, a estratégia de saúde da família é o principal modelo adotado para a personificação da intencionalidade prática e cultural desta estrutura, sendo um ponto basilar assistencial, na facilitação do acesso e redução da demanda desnecessária nos demais pontos da rede de atenção à saúde, com ênfase nas hospitalizações (PINTO LF e GIOVANELLA L, 2018).

Ainda nos dias atuais, podemos compreender a importância das abordagens que objetivam a consolidação das Redes de Atenção à Saúde, resgatando e evidenciando sobremaneira a Atenção Primária à Saúde como coordenadora e ordenadora deste processo, na busca pela integralidade do cuidado como reflexo aos mais variados problemas apresentados em cada território (GIOVANELLA L e MENDONÇA MHM, 2012). Neste arranjo assistencial, constata-se o anseio e dedicação objetivando a investigação de associações entre a frequência das internações por condições sensíveis à atenção primária com as características socioeconômicas, níveis de escolaridade, renda dentre outros (HUANG Y, et al., 2019).

As internações por condições sensíveis à atenção primária são episódios assistenciais nos quais a admissão em uma unidade hospitalar poderia ter sido evitada, sendo estas condições passíveis de abordagem em tempo oportuno quando do acesso adequado, ambulatoriamente por meio da (APS) (KIM AM, et al., 2019). Com o passar dos anos, discussões sobre a eficiência na gestão de ações e serviços públicos de saúde passaram a se apresentar como pauta necessária, agregando valores para respostas a soluções coletivas, consequentemente, melhor alocação dos escassos recursos financeiros existentes (BROUSSELE A, et al., 2011).

Sabe-se que a discussão sobre os fatores que impactam nas Internações por Condições Sensíveis a Atenção Primária (ICSAP) é controversa (NEDEL FB, et al., 2008; NEDEL FB, et al., 2010; NEDEL FB, et al., 2011; ALFRADIQUE ME, et al., 2009; FACCHINI LA, et al., 2008; ELIAS E e MAGAJEWSKI F, 2008; BRASIL, 2008). Uma revisão sistemática cujo objetivo foi identificar as relações entre a APS e as hospitalizações por condições evitáveis, ratificou que quanto maior a presença da atenção primária, menores as taxas de ICSAP (BUSBY J, et al., 2015; LOENEN TV, et al., 2014).

Ademais, considerando o senso comum na gestão municipal, de forma tendenciosa ou não, buscam-se respostas sobre o peso associado à APS pelas ICSAP, porém, raramente, avaliam a insuficiência de recursos financeiros na APS como fator impactante neste desfecho. De forma ampliada, Bastos RMR, et al. (2014) destaca a necessidade de análise que contemple fatores demográficos e regionais que poderão responder as fragilidades estruturais e processuais fundamentais para a gestão municipal. Por outro lado, estes fatores não consideram reflexos de possíveis aumentos orçamentários e gastos gerais com Ações e Serviços Públicos em Saúde (ASPS), não somente na atenção primária à saúde em cada localidade, cujos resultados favoráveis com a redução das internações por estas condições possam estar incidindo sobre o aporte orçamentário e a execução financeira em variadas frentes. 
Dado o exposto, o objetivo do presente estudo foi avaliar associações entre características socioeconômicas dos municípios e os gastos per capita com atividades de saúde e as relações com as internações por condições sensíveis à atenção primária em 50 municípios de cinco microrregiões da macrorregião Sul do Estado de Minas Gerais.

\section{MÉTODOS}

Trata-se de um estudo transversal analítico utilizando-se de dados secundários de acesso amplo. Para o levantamento das estimativas populacionais foram consideradas as tabelas disponibilizadas por meio da plataforma do Instituto Brasileiro de Geografia e Estatística (IBGE).

As categorizações quanto ao porte populacional consideraram também como referência, publicações deste mesmo instituto, relacionando a esta categoria cada um dos 50 municípios pertencentes ao objeto de estudo e suas respectivas populações. Para a identificação da tipologia de cada município foi considerada a classificação e caracterização dos espaços rurais e urbanos do Brasil/IBGE, disponibilizada pelo Departamento de Atenção Básica do Ministério da SaúdE (DAB).

Para o levantamento dos dados do PIB per capta e IDHM, foram considerados os dados dispostos por meio da plataforma denominada IBGE cidades. Os dados referentes ao coeficiente de GINI 2010 foram tabulados utilizando-se do acesso ao site do Departamento de informática do SUS com a ferramenta Tabnet (DATASUS).

Os gastos per capita foram extraídos do banco de dados do Sistema de Informação sobre Orçamentos Públicos em Saúde (SIOPS), organizados posteriormente por meio de uma planilha do software excel.

Para cálculo das taxas de ICSAP por município, foram utilizados os dados de internações tabulados no programa TabWin versão 4.1.5. Os bancos de dados contendo os arquivos reduzidos das internações hospitalares para o estado de Minas Gerais(RDMG), Autorizações de Internações Hospitalares (AIH), para a análise das referidas internações, foram atualizados para cada um dos anos de atendimento correspondentes, considerando em cada análise os municípios de residência deste estudo, assinalando ainda como incremento de análise, a frequência, nas seleções ativas considerou-se as categorizações já apresentadas pelo Datasus como definições para as condições sensíveis à atenção primária denominada "At. Bas.3".

Os dados relacionados aos beneficiários de planos privados de saúde foram extraídos do site da Agência Nacional de Saúde Suplementar (ANS). Os downloads correspondentes as tabelas nacionais e arquivos de definição foram extraídos do site do DATASUS. As variáveis de desfecho e as variáveis independentes foram dicotomizadas pelas medianas dos 50 municípios.

As variáveis quantitativas foram descritas com média (desvio padrão), mediana (valor mínimo e máximo) e as categóricas com frequências absolutas e relativas. Foram estimados modelos de regressão logística simples entre cada variável independente e os desfechos "internações por condições sensíveis em 2017" e "internações por condições sensíveis em 2018".

A seguir, as variáveis com $p<0,20$ nas análises simples (brutas) foram estudadas em modelos de regressão logística múltipla. Permaneceram no modelo final as variáveis com $p \leq 0,05$ após os ajustes. A partir dos modelos de regressão foram estimados os Odds Ratios (OR). Todas as análises foram realizadas no programa $\mathrm{R}$, com nível de significância de 5\%. Este estudo não foi submetido a Comitê de Ética em Pesquisa por utilizar dados secundários de amplo domínio público.

\section{RESULTADOS}

Na Tabela 1 é apresentada a análise descritiva das variáveis estudadas dos 50 municípios analisados. Observa-se que os municípios tiveram uma média de 23,11 e 22,68 internações por condições sensíveis em 2017 e 2018, respectivamente. Os gastos per capita em 2017 e 2018 com atividades de saúde foram de $\mathrm{R} \$ 754,82$ e $\mathrm{R} \$ 834,37$, respectivamente. 
Tabela 1 - Análise descritiva dos dados dos 50 municípios de cinco microrregiões da macroregião Sul do Estado de Minas Gerais.

\begin{tabular}{|c|c|c|}
\hline Variável & Categoria & Frequência (\%) \\
\hline \multirow{6}{*}{$\begin{array}{l}\text { Porte populacional (número de } \\
\text { habitantes) }\end{array}$} & Até 5.000 & $14(28,0 \%)$ \\
\hline & 5.001 a 10.000 & $12(24.0 \%)$ \\
\hline & 10.001 a 20.000 & $12(24,0 \%)$ \\
\hline & 20.001 a 50.000 & $8(16,0 \%)$ \\
\hline & 50.001 a 100.000 & $2(4,0 \%)$ \\
\hline & 100.001 a 500.000 hab. & $2(4,0 \%)$ \\
\hline \multirow{2}{*}{ Classificação do IDHM - 2010} & Médio & $29(58,0 \%)$ \\
\hline & Alto & $21(42,0 \%)$ \\
\hline- & Média (desvio padrão) & Mediana (valor mínimo e máximo) \\
\hline \multirow{2}{*}{$\begin{array}{l}\text { PIB per capta }-2017 \\
\text { Gasto per capta com atividades de } \\
\text { saúde }-2017\end{array}$} & $19.320,21(8.710,01)$ & $17272,77(9464,16 ; 51827,58)$ \\
\hline & $754,82(327,49)$ & $645,34(256,25 ; 1596,80)$ \\
\hline $\begin{array}{l}\text { Gasto per capta com atividades de } \\
\text { saúde }-2018\end{array}$ & $834,37(352,40)$ & $736,78(269,91 ; 1918,60)$ \\
\hline \multirow{3}{*}{$\begin{array}{l}\text { Coeficiente de GINI - } 2010 \\
\text { IDHM - } 2010 \\
\text { Proporção da população coberta por } \\
\text { convênio - } 2019\end{array}$} & $0,48(0,06)$ & $0,48(0,39 ; 0,65)$ \\
\hline & $0,70(0,03)$ & $0,70(0,64 ; 0,78)$ \\
\hline & $15,98(10,02)$ & $14,72(1,55 ; 40,34)$ \\
\hline $\begin{array}{l}\text { Internações por condições sensíveis - } \\
2017\end{array}$ & $23,11(7,30)$ & $22,64(11,99 ; 37,60)$ \\
\hline $\begin{array}{l}\text { Internações por condições sensíveis - } \\
2018\end{array}$ & $22,68(7,08)$ & $20,94(10,97 ; 38,46)$ \\
\hline
\end{tabular}

Fonte: Gonçalves E e Mialhe FL; dados extraídos dos sistemas of iciais governamentais.

Na Tabela 2 são apresentados os resultados das análises de associação com internações por condições sensíveis em 2017. Observa-se que apenas o gasto per capita com atividades de saúde em 2017 apresentou associação significativa $(p<0,05)$. Entre os munícipios que gastaram menos per capita, $64,0 \%$ tiveram número de internações acima da mediana em 2017. Já entre os municípios que gastaram mais per capita, 36,0\% tiveram internações acima da mediana. Municípios com menor gasto per capita com atividades de saúde apresentaram 3,16 vezes mais chance de ter maior número de internações por condições sensíveis $(p=0,05)$.

Tabela 2 - Análises (brutas e ajustadas) das associações com internações por condições sensíveis em 2017, para os 50 municípios de cinco microrregiões da macroregião Sul do Estado de Minas Gerais.

\begin{tabular}{|c|c|c|c|c|c|c|c|c|}
\hline \multirow{2}{*}{ Variável } & \multirow{2}{*}{ Categoria } & \multirow{2}{*}{ n (\%) } & \multicolumn{2}{|c|}{$\begin{array}{c}\text { Internações por condições } \\
\text { sensíveis em } 2017\end{array}$} & \multirow[t]{2}{*}{$\begin{array}{l}\text { PR bruto } \\
\text { ("IC95\%) }\end{array}$} & \multirow[t]{2}{*}{ p-valor } & \multirow[t]{2}{*}{ 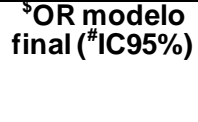 } & \multirow[t]{2}{*}{$\begin{array}{l}\text { p- } \\
\text { valor }\end{array}$} \\
\hline & & & $\begin{array}{c}{ }^{*} \text { Menor } \\
\mathrm{n}(\%)\end{array}$ & $\begin{array}{c}{ }^{*} \text { Maior } \\
\mathbf{n}(\%)\end{array}$ & & & & \\
\hline \multirow{2}{*}{ Porte } & "Menor & $26(52.0 \%)$ & $14(53.8 \%)$ & $12(46.2 \%)$ & & & - & - \\
\hline & Maior & $24(48,0 \%)$ & $11(45,8 \%)$ & $13(54,2 \%)$ & $\begin{array}{c}1,38(0,45- \\
4,20)\end{array}$ & 0,5717 & & \\
\hline \multirow{2}{*}{$\begin{array}{l}\text { Co eficiente GINI } \\
2010\end{array}$} & \#Menor & 25 (50,0\%) & $12(48,0 \%)$ & $13(52,0 \%)$ & $\begin{array}{c}1,17(0,39- \\
356)\end{array}$ & 0,7775 & - & - \\
\hline & Maior & $25(50,0 \%)$ & $13(52,0 \%)$ & $12(48,0 \%)$ & Ref & & & \\
\hline \multirow{2}{*}{ IDHM 2010} & "Menor & $29(58,0 \%)$ & $13(44,8 \%)$ & $16(55,2 \%)$ & $\begin{array}{c}1,64(0,53- \\
5,09)\end{array}$ & 0,3913 & - & - \\
\hline & Maior & $21(42,0 \%)$ & $12(57,1 \%)$ & $9(42,9 \%)$ & Ref & & & \\
\hline \multirow{2}{*}{$\begin{array}{l}\text { Gasto per capita } \\
\text { com atividades } \\
\text { de saúd e em } \\
2017\end{array}$} & "Menor & $25(50,0 \%)$ & $9(36,0 \%)$ & $16(64,0 \%)$ & $\begin{array}{c}3,16(1,00- \\
10,03)\end{array}$ & 0,05 & $\begin{array}{c}3,16(1,00- \\
10,03)\end{array}$ & 0,05 \\
\hline & Maior & $25(50,0 \%)$ & $16(64,0 \%)$ & $9(36,0 \%)$ & Ref & & Ref & \\
\hline
\end{tabular}

Legenda: Evento de desfecho. Ref: Categoria de referência para as variáveis independentes; OR: Odds ratio. IC: Intervalo de confiança. \#sMediana da amostra.

Fonte: Gonçalves E e Mialhe FL; dados extraídos dos sistemas of iciais governamentais.

Os resultados das análises de associação com o número de internações por condições sensíveis em 2018 são apresentados na Tabela 3. Nesse caso, os gastos per capita com atividades de saúde em 2017 e em 2018 apresentaram $p<0,20$ e foram testadas em um modelo múltiplo. Apenas gastos percapita com atividades de saúde em 2017 permaneceu no modelo final $(p<0,05)$. Entre os munícipios que gastaram menos per capita em 2017, 64,0\% tiveram número de internações acima da mediana em 2018. Já entre os municípios que gastaram mais per capita, 36,0\% tiveram internações acima da mediana. Municípios com menor gasto per 
capita com atividades de saúde em 2017 tiveram 3,16 vezes mais chance de ter maior número de internações por condições sensíveis em 2018 ( $p=0,05)$.

Tabela 3 - Análises (brutas e ajustadas) das associações com internações por condições sensíveis em 2018, para os 50 municípios de cinco microrregiões da macroregião Sul do Estado de Minas Gerais.

\begin{tabular}{|c|c|c|c|c|c|c|c|c|}
\hline \multirow{2}{*}{ Variável } & \multirow{2}{*}{ Categoria } & \multirow{2}{*}{ n (\%) } & \multicolumn{2}{|c|}{$\begin{array}{c}\text { Internações por condições } \\
\text { sensíveis em } 2018 \\
\end{array}$} & \multirow{2}{*}{$\begin{array}{l}\text { \$OR bruto } \\
\text { ("IC95\%) }\end{array}$} & \multirow[b]{2}{*}{$p$-valor } & \multirow{2}{*}{$\begin{array}{l}\text { \$OR modelo } \\
\text { final ("IC95\%) }\end{array}$} & \multirow{2}{*}{$\begin{array}{c}\text { p- } \\
\text { valor }\end{array}$} \\
\hline & & & $\begin{array}{c}{ }^{\#} \text { Menor } \\
\mathrm{n}(\%)\end{array}$ & $\begin{array}{c}{ }^{*} \text { Maior } \\
\mathrm{n}(\%)\end{array}$ & & & & \\
\hline Porte & $\begin{array}{l}\text { "Menor } \\
\text { Maior }\end{array}$ & $\begin{array}{l}26(52,0 \%) \\
24(48,0 \%) \\
\end{array}$ & $\begin{array}{l}14(53,8 \%) \\
11(45,8 \%) \\
\end{array}$ & $\begin{array}{l}12(46,2 \%) \\
13(54,2 \%) \\
\end{array}$ & $\begin{array}{c}\text { Ref } \\
1,38(0,45- \\
\end{array}$ & 0,5717 & - & - \\
\hline \multirow[t]{2}{*}{$\begin{array}{l}\text { Coeficiente } \\
\text { GINI } 2010\end{array}$} & \#Menor & $25(50,0 \%)$ & $13(52,0 \%)$ & $12(48,0 \%)$ & $\begin{array}{c}0,85(0,28- \\
2,58)\end{array}$ & 0,7775 & - & - \\
\hline & Maior & $25(50,0 \%)$ & $12(48,0 \%)$ & $13(52,0 \%)$ & Ref & & & \\
\hline \multirow{2}{*}{ IDHM 2010} & "Menor & $29(58,0 \%)$ & $13(44,8 \%)$ & $16(55,2 \%)$ & $\begin{array}{c}1,64(0,53- \\
5,09)\end{array}$ & 0,3913 & - & - \\
\hline & Maior & $21(42,0 \%)$ & $12(57,1 \%)$ & $9(42,9 \%)$ & Ref & & & \\
\hline \multirow{2}{*}{$\begin{array}{l}\text { Gasto per } \\
\text { capita com } \\
\text { atividades } \\
\text { de saúde } \\
\text { em } 2017\end{array}$} & \#Menor & $25(50,0 \%)$ & $9(36,0 \%)$ & $16(64,0 \%)$ & $\begin{array}{l}3,16(1,00- \\
10,03)\end{array}$ & 0,0500 & $\begin{array}{c}3,16(1,00- \\
10,03)\end{array}$ & 0,0500 \\
\hline & Maior & $25(50,0 \%)$ & $16(64,0 \%)$ & $9(36,0 \%)$ & Ref & & Ref & \\
\hline \multirow{2}{*}{$\begin{array}{l}\text { Gasto per } \\
\text { capita com } \\
\text { atividades } \\
\text { de saúde } \\
\text { em } 2018\end{array}$} & "Menor & $25(50,0 \%)$ & $10(40,0 \%)$ & $15(60,0 \%)$ & $\begin{array}{c}2,25(0,73- \\
6,98)\end{array}$ & 0,1602 & - & - \\
\hline & Maior & $25(50,0 \%)$ & $15(60,0 \%)$ & $10(40,0 \%)$ & Ref & & & \\
\hline
\end{tabular}

Legenda: Evento de desfecho. Ref: Categoria de referência para as variáveis independentes; OR: Odds ratio. IC: Intervalo de confiança. \#కMediana da amostra.

Fonte: Gonçalves E e Mialhe FL; dados extraídos dos sistemas oficiais governamentais.

Na Tabela 4 são apresentados os resultados das associações com internações por condições sensíveis em 2017, considerando a cobertura por convênios e o PIB per capita como variáveis independentes. $O$ resultado foi o mesmo apresentado acima, ou seja, municípios com menor gasto per capita com atividades de saúde em 2017 tiveram 3,16 vezes mais chance de ter maior número de internações por condições sensíveis em $2017(p=0,05)$.

Tabela 4 - Análises (brutas e ajustadas) das associações com internações por condições sensíveis em 2017, para os 50 municípios de cinco microrregiões da macroregião Sul do Estado de Minas Gerais.

\begin{tabular}{|c|c|c|c|c|c|c|c|c|}
\hline \multirow{2}{*}{ Variável } & \multirow{2}{*}{ Categoria } & \multirow{2}{*}{ n (\%) } & \multicolumn{2}{|c|}{$\begin{array}{l}\text { Internações por condições } \\
\text { sensíveis em } 2017\end{array}$} & \multirow{2}{*}{$\begin{array}{l}\text { \$OR bruto } \\
\text { ("IC95\%) }\end{array}$} & \multirow{2}{*}{ p-valor } & \multirow{2}{*}{$\begin{array}{c}\text { \$OR modelo } \\
\text { final } \\
\text { ("IC95\%) }\end{array}$} & \multirow{2}{*}{$\begin{array}{c}\mathrm{p}- \\
\text { valor }\end{array}$} \\
\hline & & & $\begin{array}{c}\text { "Menor } \\
\mathrm{n}(\%)\end{array}$ & $\begin{array}{c}{ }^{*} \text { Maior } \\
\mathrm{n}(\%)\end{array}$ & & & & \\
\hline Cobertura & "Menor & $25(50,0 \%)$ & $10(40,0 \%)$ & $15(60,0 \%)$ & $2,25(0,73-6,98)$ & 0,1602 & - & - \\
\hline $\begin{array}{l}\text { por } \\
\text { convênios }\end{array}$ & Maior & $25(50,0 \%)$ & $15(60,0 \%)$ & $10(40,0 \%)$ & Ref & & & \\
\hline $\begin{array}{l}\text { PIB per } \\
\text { capita }\end{array}$ & $\begin{array}{l}\text { \#Menor } \\
\text { Maior }\end{array}$ & $\begin{array}{l}25(50,0 \%) \\
25(50,0 \%) \\
\end{array}$ & $\begin{array}{l}11(44,0 \%) \\
14(56,0 \%) \\
\end{array}$ & $\begin{array}{l}14(56,0 \%) \\
11(44,0 \%) \\
\end{array}$ & $\begin{array}{c}1,62(0,53-4,95) \\
\text { Ref }\end{array}$ & 0,3973 & - & - \\
\hline $\begin{array}{l}\text { Coeficiente } \\
\text { GINI 2010 }\end{array}$ & $\begin{array}{l}\text { \#Menor } \\
\text { Maior }\end{array}$ & $\begin{array}{l}25(50,0 \%) \\
25(50,0 \%)\end{array}$ & $\begin{array}{l}12(48,0 \%) \\
13(52,0 \%) \\
\end{array}$ & $\begin{array}{l}13(52,0 \%) \\
12(48,0 \%) \\
\end{array}$ & $\begin{array}{c}1,17(0,39-3,56) \\
\text { Ref }\end{array}$ & 0,7775 & - & - \\
\hline IDHM 2010 & $\begin{array}{l}\text { \#Menor } \\
\text { Maior }\end{array}$ & $\begin{array}{l}29(58,0 \%) \\
21(42,0 \%)\end{array}$ & $\begin{array}{l}13(44,8 \%) \\
12(57,1 \%) \\
\end{array}$ & $\begin{array}{l}16(55,2 \%) \\
9(42,9 \%)\end{array}$ & $\begin{array}{c}, 64(0,53-5,09) \\
\text { Ref }\end{array}$ & 0,3913 & - & - \\
\hline $\begin{array}{l}\text { Gasto per } \\
\text { capita com } \\
\text { atividades }\end{array}$ & \#Menor & $25(50,0 \%)$ & $9(36,0 \%)$ & $16(64,0 \%)$ & $\begin{array}{l}3,16(1,00- \\
10,03)\end{array}$ & 0,0500 & $\begin{array}{l}3,16(1,00- \\
10,03)\end{array}$ & 0,0500 \\
\hline $\begin{array}{l}\text { de saúde } \\
\text { em } 2017\end{array}$ & Maior & $25(50,0 \%)$ & $16(64,0 \%)$ & $9(36,0 \%)$ & Ref & & Ref & \\
\hline
\end{tabular}

Legenda: Evento de desfecho. Ref: Categoria de referência para as variáveis independentes; OR: Odds ratio. IC: Intervalo de confiança. \#sMediana da amostra.

Fonte: Gonçalves E e Mialhe FL; dados extraídos dos sistemas oficiais governamentais.

Na Tabela 5 observa-se que quando se considerou a cobertura de beneficiários de planos privados e o PIB per capita como variáveis independentes o resultado para internações por condições sensíveis em 2018 se manteve, ou seja, os municípios com menor gasto per capita com atividades de saúde em 2017 tiveram 3,16 vezes mais chance de ter maior número de internações por condições sensíveis em 2018 ( $p=0,05)$. Nesse caso, nas análises brutas, a cobertura por convênio apresentou associação significativa com 
internações por condições sensíveis em 2018, porém essa variável não permanece no modelo final quando analisada em conjunto com o gasto per capita com atividade de saúde em 2017 ( $p>0,05)$.

Tabela 5 - Análises (brutas e ajustadas) das associações com internações por condições sensíveis em 2018, para os 50 municípios de cinco microrregiões da macroregião Sul do Estado de Minas Gerais.

\begin{tabular}{|c|c|c|c|c|c|c|c|c|}
\hline \multirow{2}{*}{ Variável } & \multirow[b]{2}{*}{ Categoria } & \multirow[b]{2}{*}{ n (\%) } & \multicolumn{2}{|c|}{$\begin{array}{l}\text { Internações por condições } \\
\text { sensíveis em } 2018\end{array}$} & \multirow{2}{*}{$\begin{array}{l}\text { \$OR bruto } \\
\text { ("IC95\%) }\end{array}$} & \multirow[b]{2}{*}{ p-valor } & \multirow{2}{*}{$\begin{array}{c}\text { OR } \\
\text { modelo } \\
\text { final } \\
\left({ }^{\#} \mid \mathrm{C} 95 \%\right)\end{array}$} & \multirow[b]{2}{*}{ p-valor } \\
\hline & & & $\begin{array}{c}\text { sensiv } \\
{ }^{\text {"Menor }} \\
\mathrm{n}(\%)\end{array}$ & $\begin{array}{c}\frac{\mathrm{em} 2018}{{ }^{*} \text { Maior }} \\
\mathrm{n}(\%)\end{array}$ & & & & \\
\hline \multirow{2}{*}{$\begin{array}{l}\text { Cobertura por } \\
\text { convênios }\end{array}$} & "Menor & $26(52,0 \%)$ & $9(36,0 \%)$ & $16(64,0 \%)$ & $3,16(1,00-$ & 0,0500 & - & - \\
\hline & Maior & $24(48,0 \%)$ & $16(64,0 \%)$ & $9(36,0 \%)$ & Ref & & & \\
\hline \multirow{2}{*}{ PIB per capita } & \#Menor & $25(50,0 \%)$ & $11(44,0 \%)$ & $14(56,0 \%)$ & $\begin{array}{c}1,62(0,53- \\
4,95)\end{array}$ & 0,3973 & - & - \\
\hline & Maior & $25(50,0 \%)$ & $14(56,0 \%)$ & $11(44,0 \%)$ & Ref & & & \\
\hline \multirow{2}{*}{$\begin{array}{l}\text { Coeficiente } \\
\text { GINI } 2010\end{array}$} & \#Menor & $25(50,0 \%)$ & $13(52,0 \%)$ & $12(48,0 \%)$ & $\begin{array}{l}0,85(0,28- \\
2,58)\end{array}$ & 0,7775 & - & - \\
\hline & Maior & $25(50,0 \%)$ & $12(48,0 \%)$ & $13(52,0 \%)$ & Ref & & & \\
\hline \multirow{2}{*}{ IDHM 2010} & "Menor & $29(58,0 \%)$ & $13(44,8 \%)$ & $16(55,2 \%)$ & $\begin{array}{c}1,64(0,53- \\
5,09)\end{array}$ & 0,3913 & - & - \\
\hline & Maior & $21(42,0 \%)$ & $12(57,1 \%)$ & $9(42,9 \%)$ & Ref & & & \\
\hline \multirow{2}{*}{$\begin{array}{l}\text { Gasto per } \\
\text { capita com } \\
\text { atividades de } \\
\text { saúde em } \\
2017 \\
\end{array}$} & \#Menor & $25(50,0 \%)$ & $9(36,0 \%)$ & $16(64,0 \%)$ & $\begin{array}{c}3,16(1,00- \\
10,03)\end{array}$ & 0,0500 & $\begin{array}{c}3,16 \\
(1,00- \\
10,03)\end{array}$ & 0,0500 \\
\hline & Maior & $25(50,0 \%)$ & $16(64,0 \%)$ & $9(36,0 \%)$ & Ref & & Ref & \\
\hline \multirow{2}{*}{$\begin{array}{l}\text { Gasto per } \\
\text { capita com } \\
\text { atividades de } \\
\text { saúde em } \\
2018\end{array}$} & "Menor & $25(50,0 \%)$ & $10(40,0 \%)$ & $15(60,0 \%)$ & $\begin{array}{c}2,25(0,73- \\
6,98)\end{array}$ & 0,1602 & - & - \\
\hline & Maior & $25(50,0 \%)$ & $15(60,0 \%)$ & $10(40,0 \%)$ & Ref & & & \\
\hline
\end{tabular}

Legenda: Evento de desfecho. Ref: Categoria de referência para as variáveis independentes; OR: Odds ratio. IC: Intervalo de confiança. \#sMediana da amostra.

Fonte: Gonçalves E e Mialhe FL; dados extraídos dos sistemas of iciais governamentais.

\section{DISCUSSÃo}

Por meio dos dados analisados foi possível identificar que os municípios que aplicaram menor gasto per capita com atividades de saúde em 2017 tiveram 3,16 vezes mais chance de apresentar maior número de internações por condições sensíveis em 2017 e 2018 ( $p=0,05)$. Da mesma forma, entre os municípios com menor gasto com atividades de saúde em 2017, 64,0\% tiveram mais internações por condições sensíveis em 2017 e 2018. Já entre aqueles com maior gasto, essa porcentagem foi menor (36,0\%).

As constatações no que se refere a redução, aumento ou a estabilização das ICSAP quando da identificação de uma maior cobertura da estratégia de saúde da família, não são objeto desta análise, onde, estudos de Alfradique ME, et al. (2009) ratifica que a redução da taxa de internações pode estar associada a diversos fatores, direta ou indiretamente relacionados tais quais como os fatores sociais, econômicos, renda, melhoria dos níveis sociais e a qualificação do cuidado da atenção primária. A busca pelo equilíbrio entre ef iciência e a sustentabilidade dos sistemas de saúde tem se apresentado como um desafio em todo o mundo, considerando a complexidade e heterogeneidade epidemiológica global (LIMA LD, et al., 2017; SANTOS IS, et al., 2017; BOUSQUAT A, et al., 2017; ALBUQUERQUE MV, et al., 2016).

Alguns estudos evidenciaram a redução das taxas de internações por condições sensíveis e encontraram associações com o aumento da cobertura dos serviços de saúde no decorrer dos anos, mesmo com oscilações geográficas em todo o país, assim como também a identificação da associação de maiores cargas de trabalho do profissional médico versus maiores taxas de ICSAP (MACIEL AG, et al.,2014, BUSBY J, et al., 2015, LOENEN TV, et. al., 2014). No entanto, sem qualquer probabilidade associativa quanto aos custos gerais com gastos em ações e serviços de saúde e o indicador ICSAP nos sistemas de saúde locais, estaduais e nacional.

Considera-se que uma maior alocação de recursos para a execução de ações e serviços de saúde na atenção primária seria capaz de retratar a qualificação da assistência e, compreensivelmente a atenuação da busca desnecessária dos serviços hospitalares por condições sensíveis (ALFRADIQUE ME, et al., 2009).

O presente estudo corroborou tal hipótese, pois verificou-se que os municípios com menores gastos com atividades de saúde, de forma geral e considerando uma alocação global orçamentária e financeira, tiveram 
mais internações por condições sensíveis nos dois anos analisados, e aqueles com maior gasto, essa porcentagem foi consideravelmente, menor no mesmo período. Porém, este estudo considerou os gastos gerais em ações e serviços e saúde (ASPS), sem mensurar, portanto, os pesos e alocações específicas em relação as execuções financeiras para a atenção primária à saúde. É perceptível de que sistemas de saúde estruturados a partir da APS, com uma cobertura adequada por meio das equipes de saúde da família, impactaram em menores taxas de ICSAP (SKINNER HG, et al., 2016).

Dados de Mendonça CS (2016) explicam que a vulnerabilidade socioeconômica apresenta associação em relação ao comportamento das taxas de ICSAP, significando a relevância do sistema único de saúde como indutor da inclusão social, por meio de políticas de saúde ef etivas. No entanto, não identificamos neste estudo, associações entre as internações por condições sensíveis e o índice Gini, o Índice de Desenvolvimento Humano (IDHM) e o Produto Interno Bruto (PIB) per capta.

Nos últimos anos, intencionalmente ou, de forma subjetiva tem se destacado a discussão sobre os aspectos que possivelmente, reproduzem as desconexões entre o cenário ideal e os cenários identificados quando as explicações para taxas elevadas por ICSAP (DELAMATER PL, et al., 2013). Entretanto, estudos referentes a esses custos ainda são incipientes na literatura brasileira (OLIVEIRA TL, et al., 2021).

É fundamental avaliar os diversos fatores que possam impactar os custos sobre as ICSAP no Sistema Único de Saúde onde, nos últimos anos, segundo Mendes A e Carnut $L$ (2018) novos rumos e desafios se apresentam na sustentabilidade do sistema de saúde público com o processo de desfinanciamento em curso, agravado pela Emenda Constitucional № 95, de 15 de dezembro de 2016, congelando os gastos públicos por 20 anos (FUNCIA F e SANTOS L, 2019).

Estudos nacionais de Pazó RG, et al. (2014) e internacionais Kim J, et al. (2019); Kim AM et al. (2019); Week WB et al. (2016) verificaram associações entre as taxas de ICSAP e maior disponibilidade de leitos hospitalares. Uma hipótese explicativa para esses achados é de que a maior of erta de leitos hospitalares facilitaria o acesso espontâneo ao nível terciário de atenção, induzindo a um excesso de hospitalizações, situação antagônica à busca pelo reconhecimento das reais necessidades da população, como aponta a Lei de Roemer (ROOS LL, et al., 2005). Nesse contexto, uma maior disponibilidade de leitos hospitalares podenia oportunizar a busca de atendimento em tempo oportuno, na expectativa tão somente do usuário, no sistema de saúde, refletindo ou perenizando possíveis desarranjos assistenciais, consequentemente a alocação e utilização inapropriada dos recursos e serviços de saúde (KIM J, et al., 2019).

A constatação de oscilação nas variáveis socioeconômicas e a ocorrência de ICSAP é refletida na literatura científica Kim J, et al. (2019) e Mazumdar S, et al. (2019); Dimitrovová K, et al. (2017). Estudos demonstram ainda que piores condições socioeconômicas são barreiras ao acesso a serviços, e cidadãos em estado de vulnerabilidade econômica, utilizam em menor escala os serviços de saúde e, ao utilizá-los, priorizam a busca por respostas as doenças, não considerando as ações de promoção e prevenção (KIM J, et al., 2019).

Em um país com dimensões continentais, apresentando em suma, municípios de pequeno porte populacional, os orçamentos municipais são restritos, merecendo então especial atenção para aplicação cautelosa dos finitos recursos públicos. Segundo Souza C (2002) a constatação de aspectos peculiares diversificados neste sistema descentralizado, reproduzem contextos distintos quanto à capacidade de respostas nas dimensões administrativa, técnica, política e ética pelo ente estadual e municipal.

É salutar ressaltar que a partir do Decreto o 7.508 de 28 de junho de 2011 , que regulamentou a Lei no 8.080, de 19 de setembro de 1990, para dispor sobre a organização do Sistema Único de Saúde - SUS, o planejamento da saúde, a assistência à saúde e a articulação interfederativa, por meio do protagonismo assumido pela Rede de Atenção à Saúde (RAS), importante volume dos incentivos são direcionados às ações e aos serviços da Média e Alta Complexidade Ambulatorial e Hospitalar (MAC).

Para Duarte LS, et al. (2018), esse cenário apresenta relevância, com destaque para as redes de atenção à saúde, quando da mensuração dos volumes expressivos de recursos que sã destinados, percebidos pelo processo de regionalização, no entanto, estas redes ainda se apresentam fragmentadas e fragilizadas, não 
oportunizando o protagonismo e pelo contrário, sobrecarregando e transferindo para sociedade à APS como um ponto de atenção não resolutivo, distante das premissas históricas do papel.

Ainda, segundo Pereira BLS, et al. (2019) nos últimos anos os valores oriundos dos repasses da união para o custeio de ASPS têm se apresentado com menor expressão, onde, desta forma, faz se necessário pela gestão municipal, o fortalecimento da cultura e dos processos de orçamento e planejamento que contemplem as reais necessidades de cada território, com o monitoramento e avaliação contínua de todos os cenários assistenciais vigentes.

É necessário ainda, de forma consistente, combater as rasas impressões refletidas nas práticas pela busca dos serviços de saúde em nosso cotidiano, como narrado por Giovanella L e Mendonça MHM, (2012), dos quais padecemos de concepções errôneas de uma APS seletiva, cujo rol de responsabilidades se apresenta com tecnologias incipientes, restrito para a atenção as doenças que af etam usuários em situação de pobreza e extrema pobreza, culminando pela busca de outras portas de atenção à saúde, como, as portas de urgência e emergência, prontos socorros e unidades hospitalares nas mais variadas micro e macrorregiões.

As limitações deste estudo se apresentam mediante a possibilidade de equívocos e inconsistências quando da classificação, registros ou, subregistros das internações por condições sensíveis à atenção primária em cada um dos prestadores existentes no país, e consequentemente no processamento e envio dos dados pelos estados e municípios, quando da responsabilização dos estabelecimentos sob sua gestão. Por outro lado, estas possíveis inconsistências poderão também serem observadas por municípios que possuem em sua responsabilidade, o vínculo contratual com estes prestadores.

Os dados apresentados quanto aos aspectos relacionados a execução orçamentária em ASPS possuem característica auto declaratória pelos entes da federal, sob pena da responsabilização legal pela não aplicação dos recursos mínimos constitucionais previstos.

\section{CONCLUSÃO}

Maiores gastos em ações e serviços de saúde estiveram associados a menor número de internações por condições sensíveis à atenção primária, ainda que estes gastos apresentados no estudo não tenham sido resultados de alocações somente na APS, conforme dados apresentados por meio do SIOPS. Os resultados apresentados poderão contribuir para a identificação de possíveis arranjos assistenciais substitutivos veladamente vigentes em cada território sanitário em detrimento ao protagonismo, responsabilização da atenção primária como ordenadora da rede e coordenadora do cuidado, sendo mote de novos estudos quanto ao impacto dos gastos específicos na APS portodos os entes federativos na redução das ICSAP.

\section{REFERÊNCIAS}

1. ALBUQUERQUE MV, et al. Desigualdades regionais na saúde: mudanças observadas no Brasil de 2000 a 2016. Ciência \& Saúde Coletiva, 2017;22(4): 1055-1064.

2. ALFRADIQUE ME, et al. Internações por condições sensíveis à atenção primária: a construção da lista brasileira como ferramenta para medir o desempenho do sistema de saúde (Projeto ICSAP - Brasil). Cadernos de Saúde Pública, 2009;25(6): 1337-1349.

3. BASTOS RMR, et al. Internações por condições sensíveis primária, Minas Gerais, 2000 e 2010. Re. Saúde Pública 2014;48(6): 958-967

4. BOUSQUAT A, et al. Atenção primária à saúde e coordenação do cuidado nas regiões de saúde: perspectiva de gestores e usuários. Ciência \& Saúde Coletiva, 2017; 22: 1141-1145.

5. BRASIL. Casa Civil. Presidência da República. Decreto no 7.508 de 28 de junho de 2011. 2011, Disponível em: http://www.planalto.gov.br/ccivil_03/_ato2011-2014/2011/decreto/d7508.htm. Acessado em:25 de fevereiro de 2022.

6. LBROUSSELLE A, et al. A avaliação econômica. In: Avaliação: conceitos e métodos. Rio de Janeiro: Fiocruz, 2011.

7. BUSBY J, et al. A systematic review of the magnitude and cause of geographic variation in unplanned hospital admission rates and length of stay for ambulatory care sensitive conditions. BMC Health Se rvices Research. 2015; 15(1): 324.

8. DELAMATER PL, et al. Do More Hospital Beds Lead to Higher Hospitalization Rates? A Spatial Examination of Roemer's Law. PLoS One, 2013; 8(2): e54900.

9. DIMITROVOVÁ $\mathrm{K}$, et al. Evolution and financial cost of socioeconomic inequalities in ambulatory care sensitive conditions: an ecological study for Portugal, 2000-2014. International Journal for Equity in Health, 2017;16(1): 145. 
10. DUARTE LS, et al. O processo de regionalização do SUS e a autonomia municipal no uso dos recursos financeiros: uma análise do estado de São Paulo (2009-2014). Saúde em Debate, 2018;42(116):25-37.

11. FACCHINI LA, et al. Avaliação de efetividade da Atenção Básica à Saúde em municípios das regiões Sul e Nordeste do Brasil: contribuições metodológicas. Cadernos de Saúde Pública, 2008;24(1):159-172.

12. FUNCIA F, SANTOS L. Do subfinanciamento ao desfinanciamento da saúde: descendo as escadas. Domingueira da Saúde, 2019; 4.

13. GIOVANELLA L, MENDONÇA MHM. Atenção Primária à Saúde: seletiva ou coordenadora dos cuidados. Rio de Janeiro: CEBES; 2012.

14. HUANG Y, et al. Spatial access to health care and elderly ambulatory care sensitive hospitalizations. Public Health, 2019;169: 76-83.

15. KIM AM, et al. Hospitalizations for ambulatory care sensitive conditions as an indicator of access to primary care and excess of bed supply. BMC Health Services Research, 2019;19(1):259.

16. KIM J, et al. A Spatial Analysis of Preventable Hospitalization for Ambulatory Care Sensitive Conditions and Regional Characteristics in South Korea. Asia-Pacific Journal of Public Health, 2019;31(5):422-432.

17. LIMA LD, et al. Arranjos de governança da assistência especializada nas regiões de saúde do Brasil. Revista Brasileira de Saúde Materno Infantil, 2017; 17(1):S107- S119.

18. LOENEN TV, et al. Organizational aspects of primary care related to avoidable hospitalization: a systematic revi ew. Family Practice, 2014;31(5): 502-516.

19. MACIEL AG, et al. Impacto da Estratégia Saúde da Família sobre o perfil de morbidade hospitalar em Minas Gerais. Saúde em Debate, 2014;38:319-330.

20. MAZUMDAR S, et al. Spatial clusters of chronic preventable hospitalizations (ambulatory care sensitive conditions) and access to primary care. Journal of Public Health, 2020; 42(2): e134-e141.

21. MENDES A, CARNUT L. Capitalismo contemporâneo em crise e sua forma política: o subfinanciamento e o gerencialismo na saúde pública brasileira. Saúde e Sociedade, 2018;27:1105-1119.

22. MENDES A, FUNCIA FR. O SUS e seu financiamento. In: Marques RM, Piola SF, Roa AC, organizadores. Sistema de Saúde no Brasil: organização e financiamento Brasília:MS, Departamento de Economia da Saúde, Investimento e Desenvolvimento, OPAS/OMS no Brasil; 2016.p. 139-168.

23. MENDONÇA CS. Internações por Condições Sensíveis à Atenção Primária e Qualidade da Saúde da Família em Belo Horizonte/Brasil. Tese (Doutorado) - Programa de Pós-Graduação em Epidemiologia. Universidade Federal do Rio Grande do Sul, Porto Alegre, 2016.

24. NEDEL FB, et al. Programa Saúde da Família e condições sensíveis à atenção primária, Bagé (RS). Revista de Saúde Pública, 2008;42(6): 1041-1052.

25. NEDEL FB, et al. Características da atenção básica associadas ao risco de internar por condições sensíveis à atenção primária:revisão sistemática da literatura. Epidemiologia e Serviços de Saúde, 2010;19(1):61-75.

26. NEDEL FB, et. al. Conceptual and methodological aspects in the study of hospitalizations for ambulatory care sensitive conditions. Ciência \& Saúde Coletiva, 2011;16(1): 1145-1154.

27. OLIVEIRA TL, et al. Fatores associados ao custo das internações hospitalares por doenças sensíveis à Atenção Primária no Sistema Único de Saúde. Ciência \& Saúde Coletiva,2021;26(10):4541-4552.

28. PAZÓ RG, et al. Modelagem hierárquica de determinantes associados a internações por condições sensíveis à atenção primária no Espírito Santo, Brasil. Cadernos de Saúde Pública, 2014;30(9):1891-1902.

29. PERẺIRA BLS, et al. Portaria 3992/2017: desafios e avanços para gestão dos recursos no Sistema Único de Saúde. Ver Saude Publica. 2019; 53: 58

30. PINTO LF, GIOVANELLA L. Programa à Estratégia Saúde da Família: expansão do acesso e redução das internações por condições sensíveis à atenção básica (ICSAB). Ciência \& Saúde Coletiva, 2018;23(6): 1903-1914.

31. ROOS LL, et al. Physician visits, hospitalizations, and socioeconomic status: ambulatory care sensitive conditions in a Canadian setting. Health Services Research, 2005; 40(4):1167-1185.

32. SANTOS IS, et al. Os recursos físicos de saúde no Brasil:um olhar para o futuro. In: Brasil Saúde Amanhã: dimensões para o planejamento da atenção à saúde. Rio de Janeiro:Fiocruz; 2017.

33. SANTOS HLPC, et. al. Public expenditure on hospitalizations for COVID-19 treatment in 2020, in Brazil. Revista de Saúde Pública,2021;55: 52.

34. SKINNER HG, et al. The effects of multiple chronic conditions on hospitalization costs and utilization for ambulatory care sensitive conditions in the United States: a nationally representative cross-sectional study. BMC Health Services Research, 2016;16: 77.

35. STARFIELD B. Atenção primária: equilíbrio entre necessidades de saúde, serviços e tecnologia.

36. Souza C. Governos e sociedades locais em contextos de desigualdades e de descentralização. Cien Saude Colet 2002; 7(3):431-442.

37. Brasília: UNESCO, Ministério da Saúde, 2002.

38. UNIVERSIDADE FEDERAL DO MARANHÃO (UFMA). Universidade Aberta do SUS (UNA-SUS). Redes de Atenção à Saúde: a atenção à saúde organizada em redes. 2016. Disponível em: https://ares.unasus.gov.br/acervo/html/ARES/7563/1/Redes\%20de\%20aten\%C3\%A7\%C3\%A30\%20\%C3\%A0\%20s a\%C3\%BAde\%20-

$\% 20$ A $\% 20$ aten\%C3\%A7\%C3\%A30\%20\%C3\%A0\%20sa\%C3\%BAde\%20organizada\%20em\%20redes.pdf. Acessado em:03 de jan de 2021.

39. WEEKS WB, et al. Rates of admission for ambulatory care sensitive conditions in France in 2009-2010.European Journal of Health Economics, 2016;17(4): 453-470. 